\title{
Does Comorbidity Increase the Risk of Dengue Hemorrhagic Fever and Dengue Shock Syndrome?
}

\author{
Shahid Mahmood, ${ }^{1}$ Saadia Hafeez, ${ }^{2}$ Hiba Nabeel, ${ }^{2}$ Urooj Zahra, ${ }^{2}$ and Hammad Nazeer ${ }^{3}$ \\ ${ }^{1}$ Department of Community Medicine, Gujranwala Medical College, Gujranwala, Pakistan \\ ${ }^{2}$ Fatima Jinnah Medical College, Lahore, Pakistan \\ ${ }^{3}$ Department of Infectious Diseases, Shaukat Khanum Memorial Hospital, Lahore, Pakistan
}

Correspondence should be addressed to Shahid Mahmood; shahidsethi@hotmail.com

Received 18 June 2013; Accepted 24 July 2013

Academic Editors: P. A. Nogueira and M. A. Sosa

Copyright (C) 2013 Shahid Mahmood et al. This is an open access article distributed under the Creative Commons Attribution License, which permits unrestricted use, distribution, and reproduction in any medium, provided the original work is properly cited.

\begin{abstract}
Background. Dengue fever is an emerging public health problem in Pakistan. The aim of this study was to determine the relationship between comorbid conditions in individuals suffering from dengue fever and the development of dengue hemorrhagic fever or dengue shock syndrome. Methods. In this age- and sex-matched case control study, total of 132 cases of dengue hemorrhagic fever/dengue shock syndrome and 249 randomly selected controls were recruited from two major teaching hospitals of Lahore, Pakistan. A semistructured questionnaire was used to collect data through interview and by reviewing clinical records. SPSS version 18 was utilized for statistical analysis including conditional logistic regression. Results. Odds of developing dengue hemorrhagic fever (DHF) and dengue shock syndrome (DSS) among diabetics are higher than in controls, but this association was not found statistically significant (OR. 1.26; 95\% CI. 0.78-2.03; $P=0.34$ ). Similarly, no association was observed in individuals suffering from hypertension (OR. 0.93; 95\% CI. 0.57-1.49; $P=0.76$ ). Odds of developing DHF and DSS were higher for bronchial asthma (adjusted OR. 1.34) and pulmonary tuberculosis (adjusted OR. 1.41); however $P$ values were insignificant. Conclusion. Presence of diabetes mellitus, hypertension, ischemic heart disease and bronchial asthma among patients contracted dengue fever will not increase the risk of dengue hemorrhagic fever and dengue shock syndrome.
\end{abstract}

\section{Background}

Dengue fever is an emerging public health problem prevalent mostly in tropical and subtropical regions of the world. It is an arbovirus infection transmitted through Aedes aegypti and Aedes albopictus mosquito species. Four dengue virus serotypes (DENV-1, DENV-2, DENV-3, and DENV-4) have yet been identified and are responsible for most of the clinical manifestations, ranging from asymptomatic disease to symptomatic dengue fever (DF) and dengue hemorrhagic fever (DHF). In majority of patients, infection is self-limiting, but in small proportions, the resultant dengue shock syndrome (DSS) may increase morbidity and mortality. Infection with one serotype does not give protection against other dengue viruses, yet sequential infections increase the risk of developing dengue hemorrhagic fever (DHF) and dengue shock syndrome (DSS) $[1,2]$.
Dengue is endemic in many Southeast Asian countries and Western pacific region [2]. About 2.5 billion people ( $40 \%$ of world's population) are at risk of dengue transmission. The World Health Organization (WHO) estimates that 50 to 100 million infections occur yearly, including 500,000 DHF cases and 22,000 deaths, mostly among children. Subjects who develop DHF have 3-5\% chance of death if accompanied by Dengue shock syndrome [2,3]. In Pakistan, first dengue outbreak was reported in Karachi during 1994 and sporadic cases occurred in coming years. Since environmental conditions are conducive to Aedes mosquito breeding in Pakistan, therefore dengue virus import through travelling and trade completed the disease transmission cycle. Economic and security related migration 2004 onward introduced the virus in Lahore as well. According to Punjab health department, total of 590339 suspected cases of dengue were reported in Lahore, out of which 21685 were confirmed by serology. 
The ratio of DF/DHF has not been reported; however it has been observed that $5-10 \%$ of these cases developed DHF, whereas $<5 \%$ went to Dengue Shock Syndrome. Death rate was reported officially to be less than $1 \%$. More males (68.2\%) in age group 15-45 were affected [2-4].

The factors associated with dengue transmission include demographic and societal changes like population growth, urbanization, lack of public health awareness, and appropriate disease reporting. Pathophysiology of severe clinical manifestation observed in DHF remains poorly understood, though it is believed that secondary infection put the subjects at great risk of dengue hemorrhagic fever [3]. Dengue shock syndrome is the most fatal clinical complication carrying high mortality. Although, more than $90 \%$ of dengue fever cases do not progress to the severe form of disease, there is no effective mechanism to predict the development and the prognosis of DHF/DSS in these cases. There is no clear understanding why among a big pool of dengue fever cases, only small proportion of subject develops DHF/DSS. Understanding the predictor of DHF/DSS development would provide information to identify individuals at higher risk and on the other hand, give sufficient time to clinicians for reducing dengue related morbidity and mortality [4]. It has been postulated that presence of certain comorbidities like diabetes mellitus, hypertension, chronic renal failure, bronchial asthma, and allergies might place some patients at high risk of developing DHF/DSS $[5,6]$. Information in this regard is insufficient. However, there is a pathophysiological linkage to support this postulation. Endothelial changes occurring in diabetes mellitus and allergies might trigger biological changes resulting in increased capillary fragility and vessel permeability observed in DHF patients. Exact mechanisms are, though, not clearly understood, but it has been suggested that the patients with history of allergies have constantly activated immune system and there is liberation of proinflammatory cytokines in tissues, particularly in endothelium [7]. Similarly, in type-2 diabetes mellitus, there is an activation of T-lymphocytes and release of cytokines like gamma interferon and tumor necrosis factor alpha, which ultimately increase the capillary fragility and permeability [8]. Similar physiopathological mechanism has been observed in development of dengue hemorrhagic fever and dengue shock syndrome.

Only small number of studies have reported relationship between DHF/DSS and selected comorbidities. It has been observed that individuals with allergies using steroids and diabetes mellitus were 2.5 times more likely to develop DHF [9-11], whereas association with hypertension and bronchial asthma was found insignificant. Similarly, in Puerto Rico diabetes mellitus, gastritis, COPD, and hepatitis were more prevalent among dengue hemorrhagic patients [1214]. In Pakistan, data on relationship between presence of comorbidities and development of DHF/DSS is scarce. Current study aims at filling the knowledge gap in this regard. Information thus obtained not only facilitates the clinicians to identify the subjects at higher risk of developing DHF/DSS in early stage of disease, but also used as an evidence to start an awareness campaign for public education.

\section{Methods}

In this matched case control study conducted in two major tertiary care hospitals (Jinnah and Sir Ganga Ram) of Lahore, a total of one hundred and thirty-two cases of dengue hemorrhagic fever and 249 appropriate controls were included. These controls were randomly selected from the same health facilities, as being positive for anti-dengue IgG and were matched with cases in terms of age (frequency matched within 5-years group) and sex. The required sample size was calculated using WHO software based on book of S. K. Lwanga and S. Lemeshow taking 5\% level of significance and with $80 \%$ power. A case for this study was defined as a 15-65 years male or female, who was diagnosed as dengue hemorrhagic fever (DHF) by a trained clinician using world health organization (WHO) criteria. Time frame for this study was from September to December 2011. A pretested semistructured interview form and a checklist for clinical record review were devised to collect the data. Face to face interviews were conducted in hospital settings by the researchers themselves after taking verbal informed consent. Clinical records were reviewed by medical specialists. All the responses were first transferred to paper based forms followed by its editing and analysis by using SPSS version 18. Numerical data was presented in the form of mean \pm $\mathrm{SD}$, whereas categorical data was described using frequencies and percentages. Difference of means was compared using independent $t$-test while chi-square test and Fisher exact test (where appropriate) were used to compare difference of proportions. For ordered categories, chi-square trend was used with one degree of freedom. A $P$-value of less than 0.05 was considered statistically significant. Odds ratio was used as a measure of strength of association between outcome of interest and exposures. Crude and adjusted odds ratios along with 95\% confidence interval were computed using conditional logistic regression method. Formal permission was obtained from concerned hospitals and institutional ethical review committees to conduct this study.

\section{Results}

The purpose of this case control study was to test the hypothesis that the subjects suffering from diabetes mellitus, cardiovascular diseases, bronchial asthma, tuberculosis, and chronic liver disease have higher odds of developing dengue hemorrhagic fever and dengue shock syndrome if they contract dengue virus infection. Of 132 cases, 71 (53.8\%) were males as compared to 158 (63.5\%) in controls (Table 1). On the other hand, 61 (46.2\%) and 91 (36.5\%) females were included among cases and controls, respectively. Difference based on gender was not statistically significant $\left(\chi^{2}=3.36 ; P=0.06\right)$. Majority (51.5\%) of the cases were aged between 40 and 59 years, whereas proportion of subjects aged 20-39 and more than 60 years were $35(26.5 \%)$ and $26(19.7 \%)$, respectively, (Table 1). Age difference was not found to be statistically significant.

Of 132 cases, 57 (43.2\%) had diabetes mellitus compared to $104(41.8 \%)$ controls, respectively, but this difference between them was not statistically significant $(P=0.79)$. 
TABle 1: Comorbidities in dengue hemorrhagic fever cases in comparison to control population.

\begin{tabular}{|c|c|c|c|c|c|c|c|c|}
\hline \multirow{2}{*}{ Characteristics } & \multicolumn{2}{|c|}{ Cases $(n=132)$} & \multicolumn{2}{|c|}{ Controls $(n=249)$} & \multicolumn{2}{|c|}{ Total $(n=381)$} & \multirow[t]{2}{*}{$\chi^{2}$} & \multirow{2}{*}{$P$-value } \\
\hline & $N$ & $\%$ & $N$ & $\%$ & $N$ & $\%$ & & \\
\hline \multicolumn{9}{|l|}{ Age (years) } \\
\hline$<20$ & 03 & 2.3 & 06 & 2.4 & 09 & 2.4 & \multirow{4}{*}{7.22} & \multirow{4}{*}{0.06} \\
\hline $20-39$ & 35 & 26.5 & 49 & 19.7 & 84 & 22.0 & & \\
\hline $40-59$ & 68 & 51.5 & 114 & 45.8 & 182 & 47.8 & & \\
\hline $60+$ & 26 & 19.7 & 80 & 32.1 & 106 & 27.8 & & \\
\hline \multicolumn{9}{|l|}{ Gender } \\
\hline Male & 71 & 53.8 & 158 & 63.5 & 229 & 60.1 & \multirow{2}{*}{3.36} & \multirow{2}{*}{0.06} \\
\hline Female & 61 & 46.2 & 91 & 36.5 & 152 & 39.9 & & \\
\hline \multicolumn{9}{|c|}{ Diabetes mellitus } \\
\hline Yes & 57 & 43.2 & 104 & 41.8 & 161 & 42.3 & \multirow{2}{*}{0.07} & \multirow{2}{*}{0.79} \\
\hline No & 75 & 56.8 & 145 & 58.2 & 220 & 57.7 & & \\
\hline \multicolumn{9}{|l|}{ Hypertension } \\
\hline Yes & 67 & 50.8 & 135 & 54.2 & 202 & 53.0 & \multirow{2}{*}{0.41} & \multirow{2}{*}{0.52} \\
\hline No & 65 & 49.2 & 114 & 45.8 & 179 & 47.0 & & \\
\hline \multicolumn{9}{|c|}{ Ischaemic heart disease } \\
\hline Yes & 26 & 19.7 & 44 & 17.7 & 70 & 18.4 & \multirow{2}{*}{0.23} & \multirow{2}{*}{0.62} \\
\hline No & 106 & 80.3 & 205 & 82.3 & 311 & 81.6 & & \\
\hline \multicolumn{9}{|c|}{ Bronchial asthma } \\
\hline Yes & 14 & 10.6 & 23 & 9.2 & 37 & 9.7 & \multirow{2}{*}{0.18} & \multirow{2}{*}{0.66} \\
\hline No & 118 & 89.4 & 226 & 90.8 & 344 & 90.3 & & \\
\hline \multicolumn{9}{|c|}{ Chronic liver disease } \\
\hline Yes & 12 & 9.1 & 27 & 10.8 & 39 & 10.2 & \multirow{2}{*}{0.28} & \multirow{2}{*}{0.59} \\
\hline No & 120 & 90.9 & 222 & 89.2 & 342 & 89.8 & & \\
\hline \multicolumn{9}{|c|}{ Pulmonary tuberculosis } \\
\hline Yes & 10 & 7.6 & 13 & 5.2 & 23 & 6.0 & \multirow[t]{2}{*}{0.84} & \multirow[t]{2}{*}{0.35} \\
\hline No & 122 & 92.4 & 236 & 94.8 & 358 & 94.0 & & \\
\hline
\end{tabular}

${ }^{r}$ Column percentages presented.

Although, odds of developing dengue hemorrhagic fever (DHF) and dengue shock syndrome (DSS) among diabetics are higher than in controls (Table 2), but this association was not statistically significant (OR. 1.26; 95\% CI. 0.782.03; $P=0.34$ ). Furthermore, even duration of suffering from diabetes mellitus either in cases or among controls will not increase the odds of developing sever forms of dengue illness. Similarly, no association was observed in individuals suffering from hypertension (OR. 0.93; 95\% CI. 0.57-1.49; $P=0.76)$. Nevertheless, participants with ischaemic heart disease though had higher odds (adjusted OR. 1.52) but wide confidence interval at $95 \%$ level of significance (CI. 0.85-2.73; $P=0.15)$ shows that this pattern has been observed merely by chance (Table 2). No significant association was also found among cases of DHF and DSS with Chronic liver disease $\left(\chi^{2}=0.28\right.$ at $\left.1 \mathrm{df} ; P=0.59\right)$.

Regarding respiratory tract health issues, it was found that among cases of DHF, only $12(9.1 \%)$ and $14(10.6 \%)$ were suffering from pulmonary tuberculosis and bronchial asthma compared to $5.2 \%$ and $9.2 \%$, respectively. Although, odds of developing DHF and DSS were higher for bronchial asthma (adjusted OR. 1.34) and pulmonary tuberculosis (adjusted OR. 1.41); however, this pattern could not be generalized as wide confidence intervals at $95 \%$ were observed $(0.57-3.43$ for TB and $0.62-2.88$ for asthma) with insignificant $P$-values (Table 2). In addition, duration of illnesses was not found to be statistically significant with these comorbidities $(P=0.40$ for bronchial asthma and $P=0.36$ for tuberculosis).

\section{Discussion}

Dengue infection has emerged as an important public health issue in Pakistan. Recent outbreak in Lahore during 2011 has demonstrated the fatal nature of this arbovirus infection. Large proportion of dengue seropositive patients developed severe manifestations of disease like dengue hemorrhagic fever (DHF) and dengue shock syndrome (DSS) and more than three hundred precious lives lost. Since pathophysiology of this disease is still poorly understood and there is no mechanism to predict or identify which case would develop DHF and DSS, therefore there evidence should be gathered 
TABLE 2: Risk of dengue hemorrhagic fever (DHF) in relation to existing comorbidities $(n=381)$.

\begin{tabular}{|c|c|c|c|c|c|c|}
\hline \multirow{2}{*}{ Comorbidity } & \multicolumn{3}{|c|}{ Unadjusted estimates } & \multicolumn{3}{|c|}{ Adjusted estimates ${ }^{*}$} \\
\hline & OR. & 95\% CI. & $P$ & OR. & 95\% CI. & $P$ \\
\hline \multicolumn{7}{|c|}{ Diabetes mellitus } \\
\hline No & 1 & Reference & & 1 & Reference & \\
\hline Yes & 1.05 & $0.69-1.62$ & 0.79 & 1.26 & $0.78-2.03$ & 0.34 \\
\hline \multicolumn{7}{|l|}{ Hypertension } \\
\hline No & 1 & Reference & & 1 & Reference & \\
\hline Yes & 0.87 & $0.57-1.32$ & 0.52 & 0.93 & $0.57-1.49$ & 0.76 \\
\hline \multicolumn{7}{|c|}{ Ischaemic heart disease (IHD) } \\
\hline No & 1 & Reference & & 1 & Reference & \\
\hline Yes & 1.14 & $0.66-1.95$ & 0.62 & 1.52 & $0.85-2.73$ & 0.15 \\
\hline \multicolumn{7}{|c|}{ Bronchial asthma } \\
\hline No & 1 & Reference & & 1 & Reference & \\
\hline Yes & 1.16 & $0.57-2.34$ & 0.66 & 1.34 & $0.62-2.88$ & 0.44 \\
\hline \multicolumn{7}{|c|}{ Pulmonary tuberculosis } \\
\hline No & 1 & Reference & & 1 & Reference & \\
\hline Yes & 1.48 & $0.63-3.49$ & 0.36 & 1.41 & $0.57-3.43$ & 0.44 \\
\hline \multicolumn{7}{|c|}{ Duration of diabetes Mellitus } \\
\hline$<5$ years & 1 & Reference & & 1 & Reference & \\
\hline $5-10$ years & 1.25 & $0.56-2.80$ & 0.57 & 2.76 & $0.77-9.84$ & 0.11 \\
\hline$>10$ years & 1.44 & $0.58-3.59$ & 0.42 & 1.86 & $0.55-6.26$ & 0.31 \\
\hline \multicolumn{7}{|c|}{ Duration of hypertension } \\
\hline$<5$ years & 1 & Reference & & 1 & Reference & \\
\hline $5-10$ years & 0.98 & $0.46-2.07$ & 0.96 & 0.92 & $0.29-2.91$ & 0.89 \\
\hline$>10$ years & 1.25 & $0.42-3.56$ & 0.71 & 0.73 & $0.14-3.66$ & 0.70 \\
\hline
\end{tabular}

*Adjusted for age, sex, and duration of illness.

to screen patient at early stage for monitoring and early intervention. This study was initiated on a premise that certain comorbidities might increase the risk of developing dengue hemorrhagic fever (DHF) and dengue shock syndrome (DSS). This premise was based on clinical observations communicated by clinicians at various forums. In order to test this hypothesis, patients of DHF/DSS were compared with those who only had dengue fever for presence of comorbidities like cardiovascular diseases, diabetes mellitus, chronic liver disease, chronic lung disorders and allergies. We did not find any statistical association with these comorbidities, though unadjusted estimates suggest that individuals suffering from diabetes mellitus and ischemic heart diseases have higher odds of developing DHF than the control population. Similar observation was also reported by Riaz et al. [10] that the diabetics were at higher risk of DHF than the control population. Similarly, of the 170 cases of DHF and 1175 controls in their study, Figueiredo et al. [11] found that individuals who reported allergies and taking steroids; those who had diabetes were 2.5 times at higher risk of developing DHF. Adjusted odds ratio in the mentioned study for diabetes was 2.75 (95\% CI. 1.12-6.73) and that for allergies was 1.29 (95\% CI. 0.87-1.89). In contrast, though adjusted odds ratio in our case was 1.26; however wide confidence interval (0.78-2.03) and insignificant $P$-value indicates the effect of smaller sample size used in our study. As regards hypertension and ischemic heart disease, we did not find any association and these findings were consistent with studies in Brazil and Puerto Rico [12]. Duration of illness in case of diabetes mellitus was significant $(\mathrm{OR} .=2.76$ for $5-$ 10 years of illness), yet no such relationship was observed for hypertension. Although, no statistical association was observed between chronic lung issues like tuberculosis and bronchial asthma, however, other studies did found positive relationship between allergies and DHF [11]. We cannot comment on this issue as none of our participant reported history of allergies; nevertheless considering close association between allergies and bronchial asthma, such link cannot be ignored.

Results of this study should be interpreted considering its retrospective nature, issues related to incomplete clinical data available, and relatively small control population for comparison. Moreover, results may lack external validity since the cases were recruited mainly from three major hospitals in Lahore and did not include patients in private clinics. Considering the strong evidence provided by studies in Brazil and Puerto Rico [12] about relationship between comorbidities and development of dengue hemorrhagic fever, we suggest a followup study with larger comparison group in order to screen those dengue cases with tendency to progress to dengue hemorrhagic fever. 


\section{Conclusion}

This is probably the first study in Pakistan to investigate the relationship between dengue hemorrhagic fever and selected comorbid conditions. We did not find any statistical association between dengue hemorrhagic fever and dengue shock syndrome with diabetes mellitus, hypertension, bronchial asthma, chronic lung disease, and chronic liver diseases.

\section{Conflict of Interests}

The authors declare no competing interests. There was no external funding involved for any part of this study.

\section{Authors' Contribution}

Shahid Mahmood contributed in study conception, design, data analysis and interpretation, and drafting the paper. Saadia Hafeez helped in study design, data collection, and drafting the paper. Hiba Nabeel participated in data collection, data management, and drafting tables. Urooj Zahra helped in data collection, paper revision, and final drafting. Hammad Nazeer helped in study design, interpretation of results, and revision of the paper. All the authors read and approved the final manuscript for publication.

\section{Acknowledgments}

The authors are grateful to all subjects and their relatives for sparing precious time for interview and allowing review of clinical records. The authors also would like to thank the cooperation and facilitation of all the medical and paramedical staff of Sir Ganga Ram and Jinnah Hospitals.

\section{References}

[1] World Health Organization, Dengue and Dengue Haemmorrhagic Fever, World Health Organization, Geneva, Switzerland, 2012.

[2] Centers for Disease Control (CDC), "Epidemiology of Dengue fever," 2012, http://www.cdc.gov/dengue/epidemiology/index .html.

[3] M. Malik, "Dengue prevention, control and management," in International Conference in Lahore Pakistan on Dengue Prevention and Management, 2012.

[4] J. A. Potts and A. L. Rothman, "Clinical and laboratory features that distinguish dengue from other febrile illnesses in endemic populations," Tropical Medicine and International Health, vol. 13, no. 11, pp. 1328-1340, 2008.

[5] D. J. Gubler, "Epidemic dengue/dengue hemorrhagic fever as a public health, social and economic problem in the 21st century," Trends in Microbiology, vol. 10, no. 2, pp. 100-103, 2002.

[6] L. Thomas, Y. Brouste, F. Najioullah et al., "Predictors of severe manifestations in a cohort of adult dengue patients," Journal of Clinical Virology, vol. 48, no. 2, pp. 96-99, 2010.

[7] G. N. Malavige, P. K. Ranatunga, V. G. N. S. Velathanthiri et al., "Patterns of disease in Sri Lankan dengue patients," Archives of Disease in Childhood, vol. 91, no. 5, pp. 396-400, 2006.
[8] J. G. Rigau-Pérez and M. K. Laufer, "Dengue-related deaths in Puerto Rico, 1992-1996: diagnosis and clinical alarm signals," Clinical Infectious Diseases, vol. 42, no. 9, pp. 1241-1246, 2006.

[9] O. Parkash, A. Almas, S. W. Jafri, S. Hamid, J. Akhtar, and H. Alishah, "Severity of acute hepatitis and its outcome in patients with dengue fever in a tertiary care hospital Karachi, Pakistan (South Asia)," BMC Gastroenterology, vol. 10, no. 2, article 43, 2010.

[10] M. M. Riaz, K. Mumtaz, M. S. Khan et al., "Outbreak of dengue fever in Karachi 2006: a clinical perspective," Journal of the Pakistan Medical Association, vol. 59, no. 6, pp. 339-344, 2009.

[11] M. A. A. Figueiredo, L. C. Rodrigues, M. L. Barreto et al., "Allergies and diabetes as risk factors for dengue hemorrhagic fever: results of a case control study," PLoS Neglected Tropical Diseases, vol. 4, no. 6, article e699, 2010.

[12] J. G. Rigau-Pérez and M. K. Laufer, "Dengue-related deaths in Puerto Rico, 1992-1996: diagnosis and clinical alarm signals," Clinical Infectious Diseases, vol. 42, no. 9, pp. 1241-1246, 2006.

[13] World Health Organization, "Impact of dengue," 2008, http://www.who.int/csr/disease/dengue/impact/en/index.html.

[14] World Health Organization, "Comprehensive guidelines for prevention and control of Dengue and Dengue haemorrhagic fever- Revised and expanded edition, 2011," SEARO technical publication series No. 60, http://www.searo.who.int/entity/vector_borne_tropical_diseases/documents/SEAROTPS60/en/index.html. 


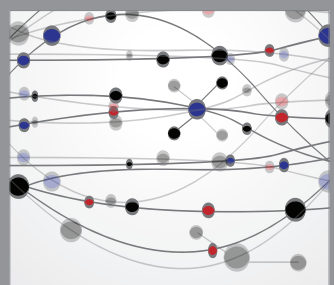

The Scientific World Journal
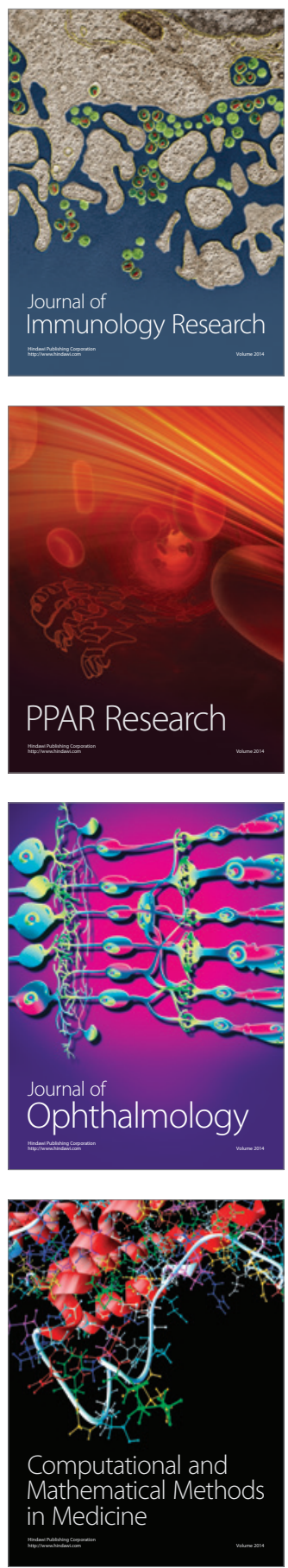

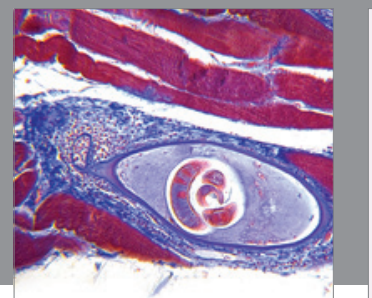

Gastroenterology

Research and Practice
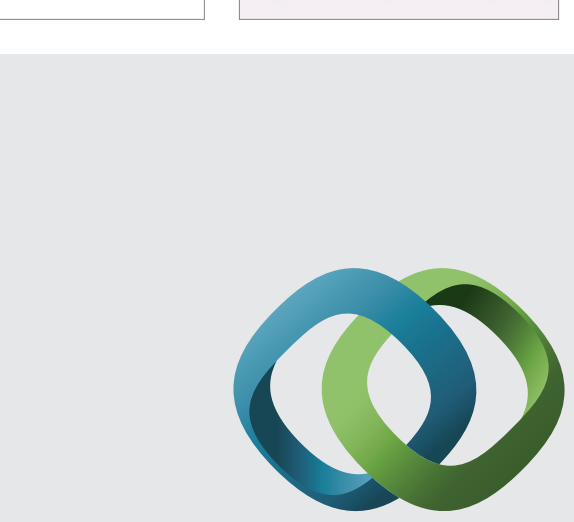

\section{Hindawi}

Submit your manuscripts at

http://www.hindawi.com
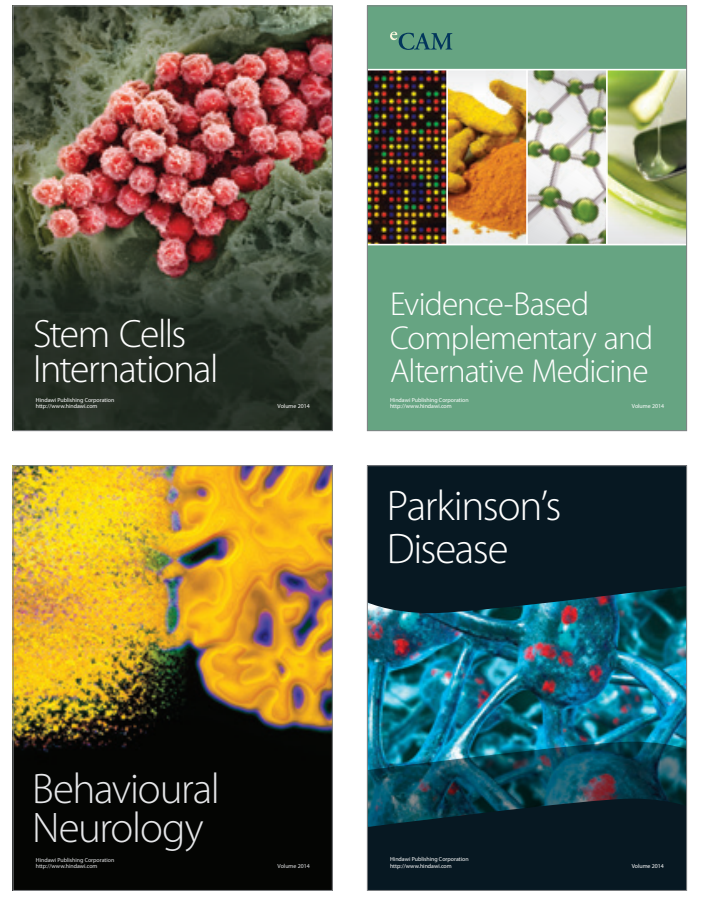
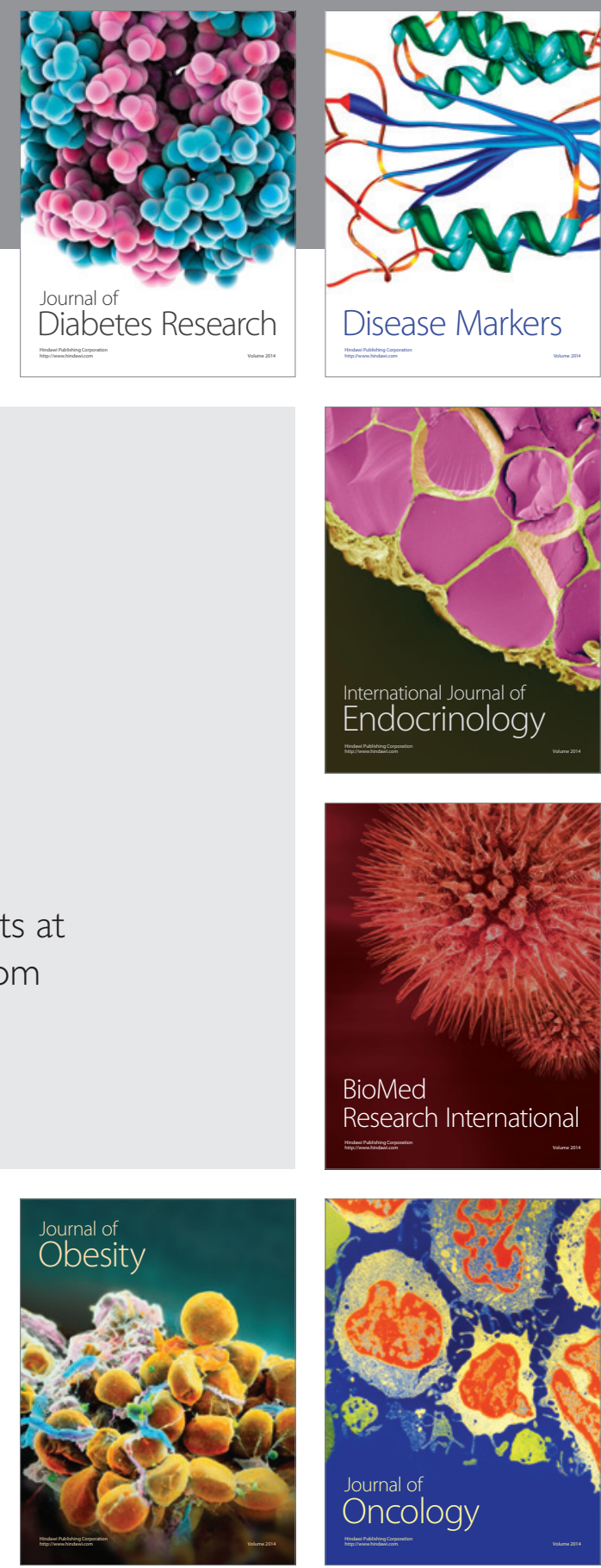

Disease Markers
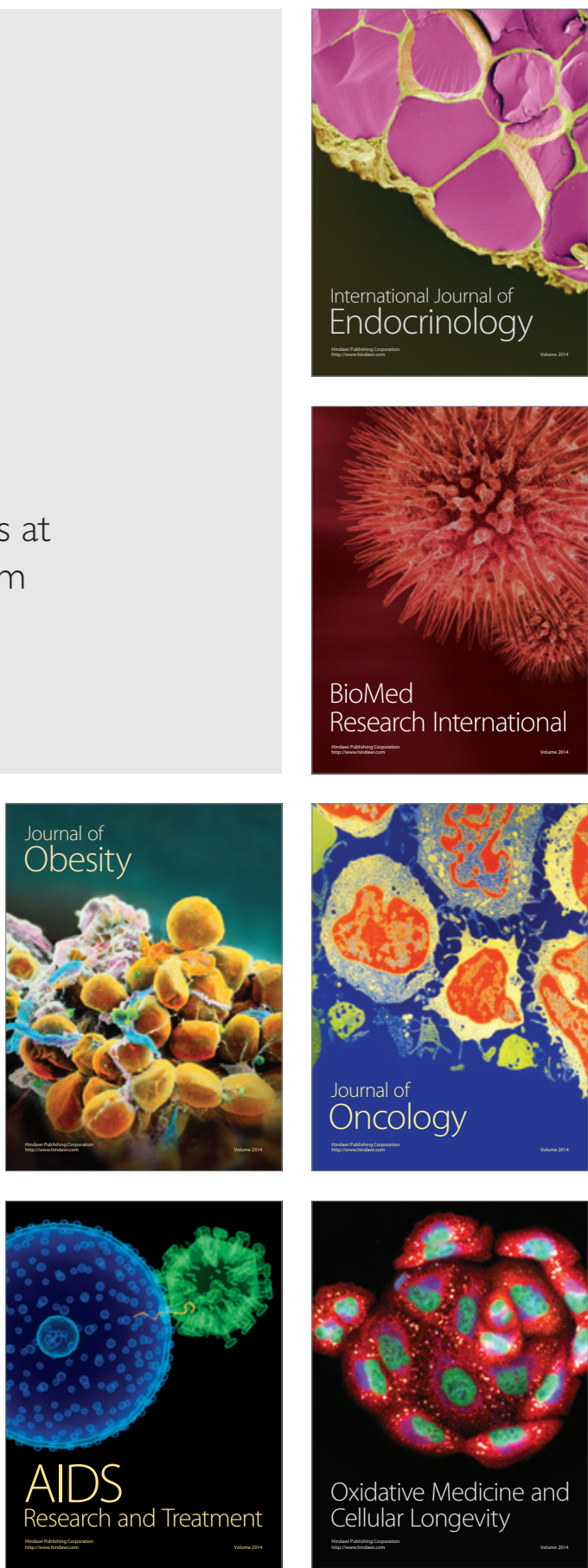